University of Nebraska - Lincoln

DigitalCommons@University of Nebraska - Lincoln

\title{
Applying Implementation Science to Support Active Collaboration in Noninvasive Brain-Computer Interface Development and Translation for Augmentative and Alternative Communication
}

Kevin Pitt

Aimee Dietz

Follow this and additional works at: https://digitalcommons.unl.edu/specedfacpub

Part of the Special Education and Teaching Commons

This Article is brought to you for free and open access by the Department of Special Education and Communication Disorders at DigitalCommons@University of Nebraska - Lincoln. It has been accepted for inclusion in Special Education and Communication Disorders Faculty Publications by an authorized administrator of DigitalCommons@University of Nebraska - Lincoln. 


\title{
Applying Implementation Science to Support Active Collaboration in Noninvasive Brain-Computer Interface Development and Translation for Augmentative and Alternative Communication
}

\author{
Kevin M. Pitt ${ }^{1}$ and Aimee Dietz ${ }^{2}$ \\ 1 Department of Special Education and Communication Disorders, \\ University of Nebraska-Lincoln, Lincoln, NE \\ 2 Department of Communication Sciences and Disorders, \\ Georgia State University, Atlanta, GA \\ Correspondence - Kevin M. Pitt: kevin.pitt@unl.edu
}

\begin{abstract}
Purpose: The purpose of this article is to consider how, alongside engineering advancements, noninvasive brain-computer interface (BCI) for augmentative and alternative communication (AAC; BCI-AAC) developments can leverage implementation science to increase the clinical impact of this technology. We offer the Consolidated Framework for Implementation Research (CFIR) as a structure to help guide future BCI-AAC research. Specifically, we discuss CFIR primary domains that include intervention characteristics, the outer and inner settings, the individuals involved in the intervention, and the process of implementation, alongside pertinent subdomains including adaptability, cost, patient needs and recourses, implementation climate,
\end{abstract}

Published in American Journal of Speech-Language Pathology 2021

doi:10.1044/2021_AJSLP-21-00152

Copyright (C) 2021 American Speech-Language-Hearing Association.

Submitted May 24, 2021; revised August 17, 2021; accepted September 27, 2021; published 27 December 2021. 
other personal attributes, and the process of engaging. The authors support their view with current citations from both the AAC and BCI-AAC fields.

Conclusions: The article aimed to provide thoughtful considerations for how future research may leverage the CFIR to support meaningful BCI-AAC translation for those with severe physical impairments. We believe that, although significant barriers to BCI-AAC development still exist, incorporating implementation research may be timely for the field of BCI-AAC and help account for diversity in end users, navigate implementation obstacles, and support a smooth and efficient translation of BCI-AAC technology. Moreover, the sooner clinicians, individuals who use AAC, their support networks, and engineers collectively improve BCI-AAC outcomes and the efficiency of translation, the sooner BCI-AAC may become an everyday tool in the AAC arsenal.

$\mathrm{N}^{\circ}$ oninvasive brain-computer interface (BCI) technology for augmentative and alternative communication (AAC), hereinafter referred to as BCI-AAC, is an interface method used to access the language content of an AAC system that does not require surgery. By providing a link between an individual's brain activity and the communication device, BCI-AAC may support communication for those with severe physical impairments by reducing access barriers to AAC software. For instance, adults with amyotrophic lateral sclerosis (ALS) or children with cerebral palsy may find currently available methods of AAC access, such as eye gaze or switch scanning, inefficient or ineffective due to paralysis and fatigue (Beukelman \& Light, 2020). Therefore, new solutions for AAC access, such as BCI-AAC, should be considered to both augment communication for those with residual speech abilities, as well as provide an alternative form of communication for those without functional speech communication (Fager et al., 2019). Unfortunately, significant barriers to BCIAAC implementation persist, as described within the Noninvasive BCI-AAC section, below. However, noninvasive BCI-AAC devices are slowly becoming a possibility for adults (Fager et al., 2019), with spelling-based P30o-based BCI-AAC systems being trialed in the homes of those with severe physical impairments (e.g., Wolpaw et al., 2018). Thus, reflecting on how noninvasive BCI-AAC technology can be developed in a manner suitable to real-world application may be timely in supporting BCIAAC access and participation for those with severe physical impairments. To this end, our article will outline how the Consolidated Framework for Implementation Research (CFIR; Damschroder et al., 2009) may be 
leveraged to help bolster multidisciplinary involvement in noninvasive BCI-AAC development, support the real-world integration of BCIAAC technology, and apply BCI-AAC to clinical populations with AAC access challenges, such as those with ALS and cerebral palsy. Through this article, we will first provide a short overview of implementation science and the CFIR. Next, we will distill key elements that clinicians must understand to implement noninvasive BCI-AAC as an AAC access option. This effort will lead to a discussion on how the primary elements of the CFIR and relevant subdomains can be harnessed to increase the clinical application of BCI-AAC.

\section{Implementation Science}

New research is largely unable to impact meaningful change without transference to the everyday practice setting, and highly supported interventions can yield reduced outcomes due to poor implementation (Moir, 2018; R. N. Rosenberg, 2003). At its foundation, implementation science supports the inclusion of multidisciplinary stakeholders in research and development to illuminate how laboratory-based research can be applied to improve care (Olswang \& Prelock, 2015). Therefore, implementation research, the term used hereon for consistency, can be described as "the scientific study of methods to promote the systematic uptake of clinical research findings and other evidence-based practices into routine practice and, hence, to improve the quality and effectiveness of health services" (e.g., Eccles \& Mittman, 2006; Ogden \& Fixsen, 2014). In more detail, implementation research forms an iterative process of improvement cycles following that the tested change may be modified, expanded, or abandoned based on real-world findings to help ensure the intervention has a meaningful impact on the intervention setting (e.g., Glasgow et al., 2012; D. H. Peters et al., 2013). For instance, while different frameworks exist (e.g., Glasgow et al., 2012) an implementation cycle may consist of plan, do, study, and act phases, during which researchers form their hypothesis, collect study data, analyze the results, and plan the next iteration, respectively (Taylor et al., 2014). To drive meaningful improvements, at the heart of this iterative process lies an intimate involvement with intervention stakeholders such as AAC professionals, individuals who use AAC, and their support network (Glasgow et al., 
2012; D. H. Peters et al., 2013). To promote this inclusion of stakeholders, implementation research commonly uses mixed-methods research including both quantitative (e.g., surveys and tools for behavioral assessment), alongside qualitative (e.g., semistructured interviews and focus groups) techniques (Bauer et al., 2015). Together, these integrative processes allow researchers to be responsive to stakeholder feedback, helping to ensure that stakeholder-sensitive and fit-for-purpose solutions are developed from the very beginning instead of waiting for late-stage research trials (Glasgow et al., 2012; Kent-Walsh \& Binger, 2018).

Multiple examples are available demonstrating the positive benefit of implementation research across a variety of areas. In this regard, Kilbourne et al. (2020) provide a review of various implementation research success stories. For instance, they described how adapting a chronic disease self-management program for different diagnoses, trialing the program with varying modalities (e.g., Internet) and racial/ethnic groups, comparing different training techniques, and partnering with community services increased the national impact of the self-management program. Closer to the field of communication sciences and disorders, Olswang and Prelock (2015) describe how their use of mixed-methods research provided valuable information necessary for improving intervention fidelity and translating their intervention aiming to teach communication signals (e.g., eye gaze) to young children with physical disabilities into a birth-to-3 center. In more detail, Olswang and Prelock acknowledge difficulties associated with implementation research such as obtaining research approval, stakeholder time commitments, and difficulties with research in the real-world environments. However, they emphasize that incorporating implementation research provided valuable information necessary for real-world implementation that would have, otherwise, remained largely concealed, such as details regarding the implementation setting, professional roles, service delivery models currently in use, costeffective procedures, and educational strategies.

Traditionally, a gap in collaboration has existed among BCI-AAC researchers, developers, and AAC stakeholders, ultimately limiting the functionality of BCI-AAC systems (Huggins \& Kovacs, 2018). Thus, even though BCI-AAC is still in the relatively early stages of development and clinical involvement, the field of implementation research 
provides a thought-provoking avenue to help ensure BCI-AAC development incorporates input from a range of stakeholders. A collaborative approach will help ensure that the engineering and development side of BCI-AAC is focused on supporting successful participation and efficient integration into clinical practice. For instance, considering implementation research can help ensure that BCI-AAC advancements are clinically helpful to a broad range of populations and that technical terminology is translated into a vernacular accessible to all stakeholders. Furthermore, implementation research can help bridge the translation gap by guiding the AAC team and BCI-AAC engineers to evaluate individuals' participation patterns and needs, along with barriers to the provision of AAC services, and how functional participation can be supported. Thus, through increased collaborative involvement and a focus on real-world applicability, implementation research may help engage a variety of individuals in BCIAAC, and help ensure future devices, procedures, and research agendas are designed to facilitate effective use across a range of contexts.

\section{CFIR}

As outlined in recent review by Nilsen (2015), various implementation frameworks exist including those focusing on (a) determinant factors that influence implementation outcomes (e.g., Damschroder et al., 2009), (b) the process of implementation (e.g., Meyers et al., 2012), and (c) factors involved in determining success (e.g., Proctor et al., 2011). For this article, we will focus on the CFIR (Damschroder et al., 2009, 2015). The CFIR provides a relevant framework for discussion as it was recently utilized by Olswang and Prelock (2015) to demonstrate implementation research applications for individuals with severe physical impairments, as described above. In addition, it provides a good foundation as it aims to consolidate a broad array of implementation research and disseminate findings in a common language suitable for BCI-AAC development. In further detail, the CFIR highlights five primary implementation domains including (a) intervention characteristics, (b) outer setting, (c) inner setting, (d) characteristics of the individuals involved, and (e) process of implementation, with each of these primary domains being composed of multiple subdomains for which Damschroder et al. (2009) encourages researchers 
to select constructs most relevant to their application. Therefore, after reviewing important foundations necessary to understand BCI$\mathrm{AAC}$ in in the next section, we will provide a thoughtful discussion regarding how primary CFIR domains and relevant subdomains can be applied to BCI-AAC.

\section{Noninvasive BCI-AAC}

A detailed explanation of noninvasive BCI-AAC methodology and performance is beyond the scope of this article; however, interested readers are referred to Pitt, Brumberg, Burnison, et al. (2019) and Brumberg, Pitt, et al. (2018). In brief, noninvasive BCI-AAC frequently requires the individual to wear an electroencephalography (EEG) cap, similar to a swimming cap, which contains recording electrodes. Commonly, gel is applied to create a link between an individual's scalp and each recording electrode, though electrodes that do not require gel are also in development (e.g., Guger et al., 2012). These electrodes record brain activity, or the summed activity of millions of neurons. Different brain signals are commonly targeted for noninvasive BCI-AAC access; such signals are related either to (a) an individual's attention to the specific stimulus they wish to select or (b) their preparation or execution of an attempted or imagined motor movement. For instance, the $\mathrm{P} 300$ is an event-related potential (brain wave) that is associated with deciding that a presented item is novel. As such, to access AAC language software via P300-based BCI-AAC, the individual focuses their attention on a target communication element such as a letter in a keyboard grid display (i.e., the novel item). At this point, all items within the grid are rapidly highlighted in a random order. As the individual focuses their attention on a specific target for selection, a positive change occurs in the EEG signal approximately $300 \mathrm{~ms}$ after the target element is highlighted. Following multiple target presentations, this $\mathrm{P} 300$ response triggers selection of the target item, commonly a letter or symbol, from the BCI-AAC interface (Donchin et al., 2000).

Technical barriers for BCI-AAC still exist that must be overcome to support clinical implementation. For instance, BCI-AAC performance may vary between different users and from day-to-day (Pitt \& Brumberg, 2021a; Shahriari et al., 2019), with muscular artifacts from uncontrolled movements or laughter negatively impacting BCI-AAC 
performance (Kögel et al., 2020; Scherer et al., 2015). Furthermore, in comparison to commercial AAC methods, BCI-AAC generally produces slower communication rates (Brumberg, Pitt, et al., 2018), and requires increased time for setup (Chavarriaga et al., 2017), in part due to the common use of wet EEG electrodes that require the application of gel for brain signal recording (Huggins \& Kovacs, 2018; Pitt, Brumberg, Burnison, et al., 2019). Finally, while research aiming to provide BCI-AAC access to children is gaining attention (e.g., Kinney-Lang et al., 2020; Pitt, Brumberg, \& Pitt, 2019; Zhang et al., 2019), existing BCI-AAC research is largely focused on providing BCIAAC access to adults with acquired impairments (Brumberg, Pitt, et al., 2018) given difficulties associated with studying the neurophysiology of children (Huggins et al., 2017). However, even taking existing BCI-AAC limitations into consideration, we believe, as BCI-AAC begins to enter trials in the home setting for adults (e.g., Wolpaw et al., 2018), that it remains imperative that stakeholders are encouraged to support the real-world implementation of BCI-AAC advancements across the life span.

\section{Application of the CFIR to BCI-AAC}

In the following section, we will discuss future directions for collaborative BCI-AAC development based on the five primary areas of the CFIR, including (a) intervention characteristics, which describes considerations regarding how BCI-AAC treatment can be designed to be a good fit across various environments; (b) the outer and (c) inner settings, two highly interrelated and overlapping areas recognizing economic, political, and social contexts; (d) the individuals involved in the intervention, considering the various people involved; and (e) the process of implementation, examining factors for moving the intervention into practice. Within each area, a selection of relevant subdomains will be discussed regarding their application to BCI-AAC (see Figure 1).

\section{Intervention Characteristics}

Adaptability. Adaptability refers to how interventions can be adapted/ tailored to meet individual needs and suit a variety of settings (e.g., 


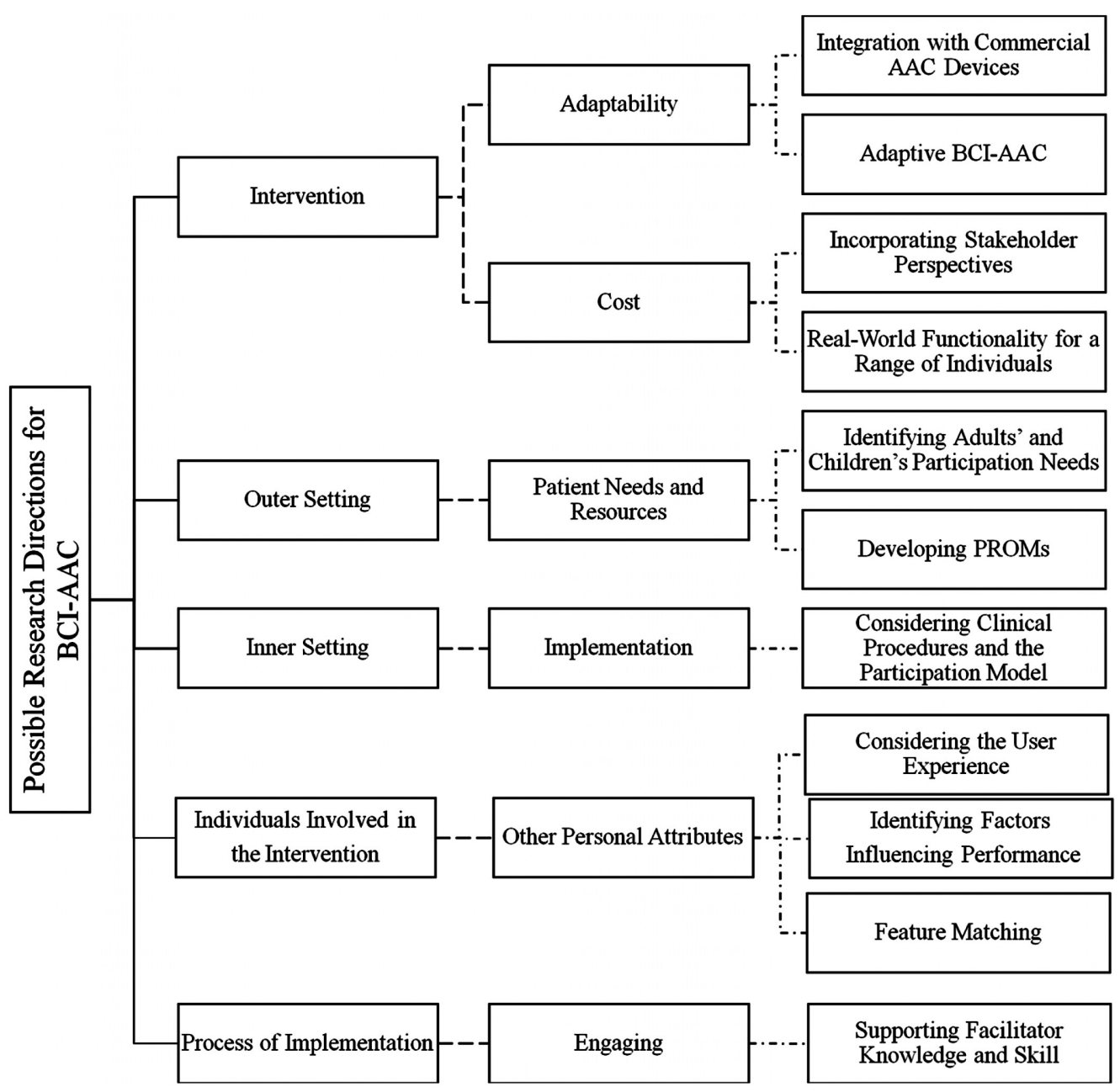

Figure 1. Possible directions for BCI-AAC research, including, going from left to right, (a) the primary CFIR domains, which are linked to the CFIR subdomains by large dashed lines, and (b) discussed areas for BCI-AAC research consideration, which are connected to CFIR subdomains by small dashed lines. BCI-AAC = braincomputer interface for augmentative and alternative communication; CFIR = Consolidated Framework for Implementation Research; PROMs = patient-reported outcome measures.

Mendel et al., 2008). AAC interventions currently seek to provide adaptability by identifying modifications that support the needs of the individuals both today and tomorrow (Beukelman \& Light, 2020). In relation to BCI-AAC, individuals who may benefit from BCI-AAC techniques demonstrate a dynamic profile due to either maturation, neurodegenerative decline, or, in some cases, recovery. However, BCI-AAC 
may currently be viewed as a last resort access method, which is primarily considered only when paralysis is severe enough to limit access by commercial AAC methods, such as eye gaze (Pitt, Brumberg, \& Pitt, 2019). This restricted view of BCI-AAC implementation likely places increased burden and anxiety on individuals who use AAC by requiring them to learn a new AAC system late in the process. Furthermore, system changes that are too abrupt increase the likelihood of device abandonment (Johnson et al., 2006), creating challenges communicating end-of-life wishes late in the disease course (in the case of neurodegeneration), impeding the ability to participate in medical decision making (in the case of recovery), or thwarting communication milestones (in the case of developmental delay). Therefore, to help support continuity and adaptability in AAC access, we value the importance of evaluating how BCI-AAC designs can adapt to integrate with conventional AAC systems. Creatively considering the use of BCIAAC to access commercial AAC systems may help support consistent communication for today and tomorrow across a range of individuals. For instance, an initial investigation demonstrated promising results for P300 BCI-AAC access to Tobii Dynavox Communicator 5 software (Gosmanova et al., 2017), with additional research currently exploring BCI-AAC access to software developed by Prentke Romich Company (Hill et al., 2021).

Furthermore, BCI-AAC techniques also show potential for supporting AAC via switch scanning for adults with ALS (e.g., Pitt \& Brumberg, 2021a) and cerebral palsy (Scherer et al., 2015). For instance, Pitt and Brumberg (2021a) demonstrated the feasibility of selecting letters from a $7 \times 5$ keyboard display via brain signals associated with imagined or attempted movements during row-column scanning for those with ALS. However, to date, studies have only focused on feasibility for single switch scanning methods.

Taken together, while research is still in the early stages, considering BCI-AAC access to traditional AAC displays may provide an avenue for an individual to maintain access to the same language software across the life span or disease course. The BCI-AAC technique that may best support continued communication access should be considered on an individual basis (e.g., Pitt \& Brumberg, 2018b; see section titled Characteristics of the Individuals Involved, below). However, as an example, an individual who utilizes a switch scanning 
access method via motor movement may wish to transition to a BCIAAC switch that is activated by an attempted or imagined movement with disease progression, or similar to existing AAC practice, consider beginning timely (early) BCI-AAC training to bolster future AAC success (Pitt \& Brumberg, 2021a). Furthermore, an individual using eye gaze may wish to transition to P300-based BCI-AAC access based on their level of impairment and preference. When considering preference, BCI-AAC use incurs a cognitive load (e.g., Pasqualotto et al., 2015); however, some individuals may find the demands for BCI-AAC access different to existing AAC methods (Brumberg, Pitt, et al., 2018). For instance, a case study report revealed that, whereas the individual with ALS found the auditory-based BCI-AAC tiring due to attentional demands, it was easier than eye gaze access as it reduced demands for precise eye movements (Käthner et al., 2015). Likewise, BCI-AAC may offer a back-up access option to those with stable, chronic conditions who battle fatigue and varying communication needs across the day. Thus, while further research is needed into how individuals experience fatigue associated with BCIAAC use throughout the day (Pitt \& Brumberg, 2021b), we believe that it is plausible that an individual may wish to have the option to alter their access methods to a single device throughout the day due to factors such as muscular fatigue and preference. Finally, current BCI-AAC systems are still largely inflexible due to their primary use as research devices (Allison, 2009). However, avenues to support adaptability are also being developed through signal processing advancements that adapt to the individual (e.g., Shenoy et al., 2006). For instance, an individual's brain signal may change between calibration and BCI-AAC use, or during BCI-AAC use due to factors such as fatigue (Talukdar et al., 2019), ultimately impairing BCI-AAC performance. Therefore, we believe that continued attention to adaptive BCI-AAC methods "learning" to adjust with an individual's changing states may support BCI-AAC outcomes (Fager et al., 2019; Lotte et al., 2013).

Cost. Cost may be expressed as the economic means to support implantation, through such avenues as funding and reimbursement (Nilsen \& Bernhardsson, 2019), and is a critical consideration for advancing BCI-AAC research and allowing for real-world implementation. BCI-AAC devices may cost around \$20,000 (Huggins \& Kovacs, 2018), and although promising and cheaper options may be available, 
more research is needed regarding their suitability for supporting dayto-day communication for those with severe physical impairments (Debener et al., 2012; Mayaud et al., 2013). Currently, BCI-AAC devices are not covered by insurance (Huggins \& Kovacs, 2018); however, we maintain that implementation research demonstrating realworld BCI-AAC functionality and the impacts of BCI-AAC technology on the lives of adults and children with physical impairments could help move BCI-AAC toward reimbursement. Furthermore, incorporating the perspectives of individuals who may use BCI-AAC (e.g., B. Peters et al., 2015) and multidisciplinary AAC professionals who have experience in AAC funding in BCI-AAC development may shed new light on barriers and solutions regarding re-imbursement.

\section{The Outer Setting}

Patient needs and resources. This subdomain addresses how patient needs, as well as barriers to meeting those needs, are known and prioritized. As the perspectives of BCI-AAC stakeholders are at the heart of driving implementation (Glasgow et al., 2012), we believe that considering the communication patterns and needs for an inclusive range of individuals is a crucial first step in guiding how BCIAAC methods can be applied to support functional communication. This consideration is consistent with user-centered design, which focuses on how designs can meet the needs and requirements of individuals who may use BCI-AAC (Kübler et al., 2014). In addition, without initially establishing person- and family-centered goals, it is difficult to identify functional outcomes for BCI-AAC. Currently, AAC professionals' caseloads are commonly composed of a heterogenous spectrum of adults and children with complex communication needs. These unique individuals have a variety of needs to bolster their quality of life and social participation. Exploring the participation needs of a range of individuals, including both adults and children, may, therefore, help elucidate the role of BCI-AAC in the clinical setting to maximize its overall impact. For instance, successful integration of BCI-AAC into clinical practice requires evaluation of individuals' natural speech abilities (Beukelman \& Light, 2020). Because individuals who use AAC devices may still possess a level of natural speech ability, we propose that BCIAAC professionals 
should not rule out the applications of BCI-AAC to support or augment the communication efforts of those who may have some natural speech ability. For example, recent studies demonstrate positive outcomes for P30o-based BCI-AAC use by individuals with cervical spinal cord injury (Ikegami et al., 2011), who may retain verbal abilities but struggle with text input to computer devices (Pouplin et al., 2016). Similarly, a person with cerebral palsy may have verbal ability sufficient for communicating with familiar communication partners but not with unfamiliar people. Text input to a computer is also important for providing access to e-mail, social media, and patient portals that may help support individuals' involvement in their medical care-especially in an expanding age of telehealth visits (Tohidast et al., 2020) - and online social participation. BCI-AAC spelling displays may provide text entry to online/social media applications (e.g., Warren \& Randolph, 2019); thus, the notion of how BCI-AAC can bolster the participation for populations who struggle with communication in certain contexts, even if they retain verbal abilities, requires continued attention.

Establishing an inventory of needs and wishes for a variety of individuals who may possibly benefit from BCIAAC may also help focus BCI-AAC development and help inform outcome measures (Andresen et al., 2016). A recent special edition from Perspectives of the ASHA Special Interest Groups titled Putting Research Into Practice highlighted how the development of patient-reported outcome measures (PROMs) may also help shed light on patient wishes (Yorkston \& Baylor, 2019). More specifically, the use of PROMs is encouraged by the International Classification of Functioning, Disability and Health to help us understand a full range of factors surrounding communication disorders and interventions (Yorkston \& Baylor, 2019). Such factors include (a) functioning at the level of the body part, (b) the whole person, and (c) the whole person within a social context, accounting for both personal and environmental factors (World Health Organization, 2002). PROMs are an important component in demonstrating the impact of an intervention (Yorkston \& Baylor, 2019) and may help decrease AAC device abandonment by supporting a match between AAC technology and the wishes, expectations, and needs of the individual. To date, while a variety of PROMs exist, none have been developed specifically for AAC (Broomfield et al., 2019); 
moreover, current PROMs do not adequately capture the values of individuals with severe physical impairments (Andresen et al., 2016). Therefore, to ensure the values of those with severe physical impairments are considered, there is a need for future research to elucidate what outcomes are most important to those who use AAC. In a BCIAAC context, the development of PROMs is still in the early stages (Andresen et al., 2016), though the importance of obtaining feedback from people who use BCIAAC is increasingly documented (e.g., BlainMoraes et al., 2012; Geronimo et al., 2015; Huggins et al., 2011; Kögel et al., 2020; B. Peters et al., 2015; Pitt \& Brumberg, 2021b). For instance, to lay the foundation for the development of PROMs, a study by Andresen et al. (2016) identified a range of domains important to those with physical disability that are not clearly captured by current tools, including those related to quality of life (i.e., social participation and opportunity, communication, roles, relationships, emotions and attitudes, environment, and physical health), and assistive technology (i.e., function, design, and support). In addition, they identified topics extremely important to those who may potentially use BCI-AAC, such as caregiver support, effort, ease of BCI setup, family, and household role. Furthermore, works such as Huggins et al. (2011) have evaluated the perspectives of those with severe physical impairments regarding outcomes for BCIAAC design, providing guidelines regarding acceptable levels of accuracy, communication rate, and setup duration.

Taken together, it may be beneficial to consider the development of PROMs for both adults and children with complex communication needs alongside quantitative performance measures for $\mathrm{AAC}$ and $\mathrm{BCI}-$ AAC, such as those related to performance accuracy, communication rate (Higginbotham et al., 2007; Thompson et al., 2014), and language use (Hill et al., 2015). These measures may provide crucial directions for BCI-AAC development and research by shedding new light on factors important to individuals who may use BCI-AAC. Furthermore, PROM developments may help standardize BCI-AAC procedures (Andresen et al., 2016; Pitt, Brumberg, \& Pitt, 2019), empower patients to be involved in decision making surrounding their care, and elucidate goals for intervention and future research (Broomfield et al., 2019; Douglas \& Burshnic, 2018). 


\section{Inner Setting}

Implementation climate: Compatibility. When considering current practice for AAC implementation, the Participation Model (Beukelman \& Light, 2020; S. Rosenberg \& Beukelman, 1987) is a commonly utilized framework for AAC assessment and treatment planning (Light \& McNaughton, 2015) for which BCI-AAC fits. The model presents a strengths-focused framework that is based on the principle that all individuals with complex communication needs can enhance their communicative abilities using AAC tools and techniques. Accordingly, the model includes evaluation of an individual's participation patterns, as well as opportunity, capability, and access barriers, along with related interventions. Opportunity barriers focus on factors that surround the individual using AAC, such as legislative and regulatory policies, common practice procedures, facilitator skill and knowledge, along with the attitudes toward individuals with disability, AAC, and participation. Furthermore, capability and access barriers identify elements associated with the individual who may use AAC, such as their current communication, natural abilities, environment, and cognitive- linguistic, sensory, motor, and literacy capabilities in relation to the operational requirements of the AAC systems. The Participation Model uses a dynamic process to guide AAC assessment and intervention that accounts for an individual's current and anticipated communication needs and preferences (Beukelman \& Light, 2020). Therefore, we propose that multidisciplinary research around the Participation Model framework can help elucidate the general context for BCI-AAC implementation in the clinical setting, maximizing the integration of BCI-AAC strategies in clinical care, and help support individuals with complex communication needs achieve communicative competence. Furthermore, beyond the Participation Model framework, the incorporation of various stakeholder perspectives as aforementioned in BCIAAC development may help shed new light on how BCI-AAC can effectively integrate with existing procedures for AAC practice.

\section{Characteristics of the Individuals Involved}

Other personal attributes. In relation to AAC, a poor fit among the person's cognitive and/or physical abilities, their environment, and the 
AAC system increases the likelihood that an individual will abandon use of their AAC device (Johnson et al., 2006). In a similar manner, prior implementation research has shown that personal attributes, such as computer experience, influenced individuals' acceptance of microcomputer technology (Frambach \& Schillewaert, 2002; Igbaria, 1993). A parallel finding was also identified by Burde and Blankertz (2006), who found that comfort with technology influenced BCI-AAC performance. Therefore, when considering the broad range of heterogenous individuals who may potentially benefit from BCI-AAC, it is important for future BCI-AAC development to reflect on how personcentered factors impact BCI-AAC performance and understand how an individual's strengths are best matched to BCI-AAC technology (Saha et al., 2021). Clinically, AAC professionals often employ a featurematching process (Beukelman \& Light, 2020; Pitt \& Brumberg, 2018b) to make clinical decisions regarding one's best AAC fit by evaluating the individual's environment, preferences, and cognitive- linguistic, sensory, motor, and literacy strengths in relation to AAC techniques. Feature-matching procedures include evaluating the holistic experience of individuals with impairment during AAC trials (e.g., levels of fatigue, effort, frustration, and satisfaction) alongside overall performance accuracy, an area that to date has received minimal incorporation into BCI-AAC research (Lorenz et al., 2014; B. Peters et al., 2016; Pitt \& Brumberg, 2021b). For instance, to help individuals with visual impairments, communication items may require increased color contrast or presentation in the auditory or tactile modality. In a parallel manner, cognitive-linguistic, sensory, motor, and literacy strengths may also impact BCIAAC performance (Fried-Oken et al., 2013; Pitt \& Brumberg, 2018b). For instance, like eye gaze access, an individual with decreased oculomotor (eye) control may benefit from communication items being in areas of the display matching their oculomotor strengths (Brumberg, Nguyen, et al., 2018). Furthermore, individuals with visual impairments may benefit from BCI-AAC access using auditory stimuli (Käthner et al., 2015). Finally, BCI-AAC methods may incur a high level of cognitive load during extended use, meaning BCI-AAC techniques are not a one-size-fits-all solution for AAC (Fager et al., 2019). Beyond intrinsic factors, by assisting with crucial areas such as device implementation and troubleshooting, the importance of the extrinsic factors, such as the support network available 
in one's environment, should not be overlooked in facilitating AAC success. However, while a foundational feature-matching framework that outlines both intrinsic and extrinsic factors impacting BCIAAC outcomes is available (Pitt \& Brumberg, 2018a, 2018b), how a range of factors impacts BCI-AAC success for those with severe physical has not been comprehensively evaluated (Chavarriaga et al., 2017; Saha et al., 2021).

Therefore, to help ensure an effective match between individuals and the emerging BCI-AAC technology, we believe it is important to explore how an individual's preferences, experiences, capabilities, and environment influence BCI-AAC outcomes. Furthermore, to support the implementation of BCI-AAC assessment in the clinical setting, it is important to develop tools that are tailored for completion by those with severe physical impairments (Fried-Oken et al., 2013; B. Peters et al., 2016; Pitt \& Brumberg, 2018a). For instance, a recent screening protocol aiming to guide cognitive-sensory-motor assessment across a range of different BCI-AAC devices was developed by Pitt and Brumberg (2018a) and was shown to be feasible for completion by those with severe physical impairments via a reliable form of yes/no responses. Furthermore, foundational tools for assessing the user experience have recently been implemented for people with physical disabilities (e.g., B. Peters et al., 2016; Pitt \& Brumberg, 2021b). However, though the development of implementation tools for BCIAAC remains in the early stages (Fried-Oken et al., 2013; B. Peters et al., 2016; Pitt \& Brumberg, 2018a, 2021b), we believe this does not preclude the integration of BCI-AAC assessment procedures into everyday practice to inform their adaptation and development.

\section{The Process of Implementation}

Engaging. As individuals' prior experience and comfort with technology tends to positively affect BCI-AAC performance (Burde \& Blankertz, 2006), as with existing AAC practice, facilitator knowledge and skill in AAC setup and use are crucial to support AAC success (Beukelman \& Light, 2020). Supporting facilitator knowledge and skill may be especially important for BCI-AAC due to technical factors such as placement of the electrode cap and ensuring a good connection between EEG electrodes and an individual's scalp (Geronimo \& 
Simmons, 2020). Therefore, how to increase individuals' knowledge, skill, and comfort with BCI-AAC implementation is likely an integral step in supporting engagement and clinical implementation of this technology. To address this issue, recent investigations have begun to document the feasibility of providing remote support for caregivers during home-based BCI-AAC implementation (Wolpaw et al., 2018) along with promising effects of providing BCI-AAC trainings via telemedicine (Geronimo \& Simmons, 2020), an especially important consideration during the COVID-19 pandemic. In addition, BCI-AAC tutorials and just-in-time training (JITT) videos will likely be critical to developing competency with BCI-AAC methodology, empowering clinicians and stakeholders to trial BCI-AAC devices, and ultimately moving this access method into everyday clinical practice. Ideally, these JITT tutorials (e.g., Brumberg, Pitt, et al., 2018; Pitt, Brumberg, Burnison, et al., 2019) will incorporate layman and clinical terms when discussing important BCI-AAC concepts for clinical application, which will help various AAC professionals to put concepts into context and develop the requisite skills to incorporate BCI-AAC into clinical practice. JITT may also be used with stakeholder groups as they work to implement the technology at home, with no clinical support. For example, to support BCI-AAC implementation, short and easily accessible training videos may be produced that outline EEG setup procedures, steps for device customization, and troubleshooting tips. In all these instances of training and support, it is also important that feedback is iteratively solicited from individuals both providing and receiving the intervention so modifications can be made to facilitate implementation and success (Douglas \& Burshnic, 2018; Douglas et al., 2015).

\section{Clinical Implications and Future Directions}

This article has focused on supporting BCI-AAC access for adults and children with severe physical impairments. However, we believe future research should build upon this work to support BCI-AAC development and translation for those with a variety of diagnose. For instance, while research is still in the early stages, noninvasive BCI-AAC techniques may promote rehabilitation outcomes for individuals with aphasia (Kleih et al., 2016) and dementia (da Silva-Sauer et al., 2019). 
Therefore, proactively applying implementation research for a broad range of individuals may further help bolster the real-world impacts of BCI-AAC technology.

In closing, it is important to consider that multiple barriers to BCIAAC development still exist (e.g., Huggins \& Kovacs, 2018) and many aspects regarding BCI-AAC design must be finalized. Basic science-focused research is crucially required to help overcome these existing barriers through avenues such as elucidating the neural mechanisms underlying BCI-AAC control, bolstering accuracy, and decreasing performance variability. Realistic expectations are important for supporting AAC success; thus, it is important that $\mathrm{AAC}$ professionals remain aware of obstacles to BCI-AAC development and have realistic expectations regarding BCI-AAC technology. While continued innovation is important, we also believe that the field can take steps now toward considering BCI-AAC research implementation. For example, we must support basic science to ensure that there are meaningful changes for those who need it most. We must also examine implementation frameworks that promote research application, engage stakeholders, and account for the diversity of people who may benefit from BCI-AAC. Moreover, as BCI-AAC devices move into home trials, we believe that considering iterative implementation frameworks is timely for helping navigate obstacles and supporting the smooth and efficient translation of BCI-AAC technology into practice. Therefore, even though forming research-clinical partnerships can be difficult (Stevens et al., 2020), and there are hurdles to performing implementation research (Moir, 2018; Olswang \& Prelock, 2015), we believe that combining important engineering and basic science advancements with implementation frameworks such as the CFIR can help ensure that BCI-AAC designs are functional and facilitate collaborations that actively engage a variety of BCI-AAC stakeholders. We hope that this article provided helpful guidance that may support multidisciplinary collaborations and future research aiming to integrate BCI-AAC as a viable AAC access option-sooner, rather than later. 
Acknowledgments This work was supported by the Nebraska Tobacco Settlement Biomedical Research Development Fund. The authors would like to thank Marj McKinty and Hannah Smith for their contributions to editing, and Kiley Allgood for figure assistance.

Declaration The authors affirm that no competing financial or nonfinancial interests existed at the time of publication.

\section{References}

Allison, B. (2009). The I of BCIs: Next generation interfaces for brain-computer interface systems that adapt to individual users. In J. A. Jacko (Ed.), Humancomputer interaction: Novel interaction methods and techniques (Vol. 2, pp. 558-568). Springer. https://doi.org/10.1007/978-3-642-02577-8_61

Andresen, E., Fried-Oken, M., Peters, B., \& Patrick, D. (2016). Initial constructs for patient-centered outcome measures to evaluate brain-computer interfaces. Disability and Rehabilitation: Assistive Technology, 11(7), 548-557. https://doi. org/10.3109/17483107.2015.1027298

Bauer, M. S., Damschroder, L., Hagedorn, H., Smith, J., \& Kilbourne, A. M. (2015). An introduction to implementation science for the non-specialist. BMC Psychology, 3(1), 32-12. https://doi.org/10.1186/s40359-015-0089-9

Beukelman, D. R., \& Light, J. C. (2020). Augmentative and alternative communication: Supporting children and adults with complex communication needs (5th ed.). Brookes.

Blain-Moraes, S., Schaff, R., Gruis, K. L., Huggins, J. E., \& Wren, P. A. (2012). Barriers to and mediators of brain-computer interface user acceptance: Focus group findings. Ergonomics, 55(5), 516-525. https://doi.org/10.1080/00140139 .2012 .661082

Broomfield, K., Harrop, D., Judge, S., Jones, G., \& Sage, K. (2019). Appraising the quality of tools used to record patient-reported outcomes in users of augmentative and alternative communication (AAC): A systematic review. Quality of Life Research, 28, 2669-2683. https://doi.org/10.1007/ s11136-019-02228-3

Brumberg, J. S., Nguyen, A., Pitt, K. M., \& Lorenz, S. D. (2018). Examining sensory ability, feature matching and assessment-based adaptation for a braincomputer interface using the steady-state visually evoked potential. Disability and Rehabilitation: Assistive Technology, 14(3), 241-249. https://doi.org/10.10 80/17483107.2018.1428369

Brumberg, J. S., Pitt, K. M., Mantie-Kozlowski, A., \& Burnison, J. D. (2018). Braincomputer interfaces for augmentative and alternative communication: A tutorial. American Journal of Speech-Language Pathology, 27(1), 1-12. https:// doi.org/10.1044/2017 ajslp-16-0244 
Burde, W., \& Blankertz, B. (2006). Is the locus of control of reinforcement a predictor of brain-computer interface performance? In G. Müller-Putz, C. Brunner, R. Leeb, R. Scherer, A. Schlögl, S. Wriessnegger, \& G. Pfurtscheller (Eds.), Proceedings of the 3rd international brain-computer interface workshop and training course (pp. 108-109). Verlag der Technischen Universität Graz.

Chavarriaga, R., Fried-Oken, M., Kleih, S., Lotte, F., \& Scherer, R. (2017). Heading for new shores! Overcoming pitfalls in BCI design. Brain-Computer Interfaces, 4(1-2), 6o-73. https://doi.org/10.1080/2326263x.2016.1263916

Damschroder, L., Aron, D. C., Keith, R. E., Kirsh, S. R., Alexander, J. A., \& Lowery, J. C. (2009). Fostering implementation of health services research findings into practice: A consolidated framework for advancing implementation science. Implementation Science, 4(1), 50. https://doi.org/10.1186/1748-5908-4-50

Damschroder, L., Hall, C., Gillon, L., Reardon, C., Kelley, C., Sparks, J., \& Lowery, J. (2015). The Consolidated Framework for Implementation Research (CFIR): Progress to date, tools and resources, and plans for the future. Implementation Science, 10(S1), A12. https://doi.org/10.1186/1748-5908-10-S1-A12

da Silva-Sauer, L., Torre-Luque, A., Silva, J. S. C., \& Fernández-Calvo, B. (2019). New perspectives for cognitive rehabilitation: Could brain-computer interface systems benefit people with dementia? Psychology \& Neuroscience, 12(1), 2537. https://doi.org/10.1037/pneoooo154

Debener, S., Minow, F., Emkes, R., Gandras, K., \& de Vos, M. (2012). How about taking a low-cost, small, and wireless EEG for a walk? Psychophysiology, 49(11), 1617-1621. https://doi.org/10.1111/j.1469-8986.2012.01471.X

Donchin, E., Spencer, K. M., \& Wijesinghe, R. (2000). The mental prosthesis: Assessing the speed of a P30o-based brain-computer interface. IEEE Transactions on Rehabilitation Engineering, 8(2), 174-179. https://doi. org/10.1109/86.847808

Douglas, N. F., \& Burshnic, V. L. (2018). Implementation science: Tackling the research to practice gap in communication sciences and disorders. Perspectives of the ASHA Special Interest Groups, 4(1), 3-7. https://doi. org/10.1044/2018_PERS-ST-2018-0000

Douglas, N. F., Campbell, W. N., \& Hinckley, J. J. (2015). Implementation science: Buzzword or game changer? Journal of Speech, Language, and Hearing Research, 58(6), S1827-S1836. https://doi.org/10.1044/2015 JSLHR-L-15-0302

Eccles, M. P., \& Mittman, B. S. (2006). Welcome to implementation science. Implementation Science, 1, 1. https://doi.org/10.1186/1748-5908-1-1

Fager, S. K., Fried-Oken, M., Jakobs, T., \& Beukelman, D. R. (2019). New and emerging access technologies for adults with complex communication needs and severe motor impairments: State of the science. Augmentative \& Alternative Communication, 35(1), 13-25. https://doi.org/10.1080/07434618.2 018.1556730

Frambach, R. T., \& Schillewaert, N. (2002). Organizational innovation adoption: A multi-level framework of determinants and opportunities for future research. Journal of Business Research, 55(2), 163-176. https://doi.org/10.1016/ $\underline{\text { S0148-2963(00)00152-1 }}$ 
Fried-Oken, M., Mooney, A., Peters, B., \& Oken, B. (2013). A clinical screening protocol for the RSVP Keyboard brain-computer interface. Disability and Rehabilitation: Assistive Technology, 10(1), 11-18. https://doi.org/10.3109/1748 3107.2013.836684

Geronimo, A., \& Simmons, Z. (2020). TeleBCI: Remote user training, monitoring, and communication with an evoked-potential brain-computer interface. Brain-Computer Interfaces, 7(3-4), 57-69. https://doi.org/10.1080/23262 63x.2020.1848134

Geronimo, A., Stephens, H. E., Schiff, S. J., \& Simmons, Z. (2015). Acceptance of brain-computer interfaces in amyotrophic lateral sclerosis. Amyotrophic Lateral Sclerosis and Frontotemporal Degeneration, 16(3-4), 258-264. https:// doi.org/10.3109/21678421.2014.969275

Glasgow, R. E., Vinson, C., Chambers, D., Khoury, M. J., Kaplan, R. M., \& Hunter, C. (2012). National Institutes of Health approaches to dissemination and implementation science: Current and future directions. American Journal of Public Health, 102(7), 1274-1281. https://doi.org/10.2105/AJPH.2012.300755

Gosmanova, K. A., Carmack, C. S., Goldberg, D., Fitzpatrick, K., Zoltan, B., Zeitlin, D. M., Wolpaw, J. R., Maehle, O. A., Borge, A., \& Vaughan, T. M. (2017). EEGbased brain-computer interface access to Tobii Dynavox Communicator 5 [Paper presentation]. Rehabilitation Engineering and Assistive Technology Society of North America. Retrieved April 22, 2021, from https://www.resna.org/sites/ default/files/conference/2017/cac/Gosmanova.html

Guger, C., Krausz, G., Allison, B. Z., \& Edlinger, G. (2012). Comparison of dry and gel based electrodes for $\mathrm{P} 300$ brain-computer interfaces. Frontiers in Neuroscience, 6, 6o. https://doi.org/10.338g/fnins.2012.0006o

Higginbotham, D. J., Shane, H., Russell, S., \& Caves, K. (2007). Access to AAC: Present, past, and future. Augmentative and Alternative Communication, 23(3), 243-257. https://doi.org/10.1080/07434610701571058

Hill, K., Huggins, J., \& Woodworth, C. (2021). Interprofessional practitioners' opinions on features and services for an augmentative and alternative communication brain-computer interface device. $P M \& R$, 13(10), 1111-1121. https://doi.org/10.1002/pmrj.12525

Hill, K., Kovacs, T., \& Shin, S. (2015). Critical issues using brain-computer interfaces for augmentative and alternative communication. Archives of Physical Medicine and Rehabilitation, 96(3), S8-S15. https://doi.org/10.1016/j. apmr.2014.01.034

Huggins, J. E., Guger, C., Ziat, M., Zander, T. O., Taylor, D., Tangermann, M., SoriaFrisch, A., Simeral, J., Scherer, R., Rupp, R., Ruffini, G., Robinson, D. K. R., Ramsey, N. F., Nijholt, A., Müller-Putz, G., McFarland, D. J., Mattia, D., Lance, B. J., Kindermans, P.-J., . . A Aernoutse, E. J. (2017). Workshops of the sixth international brain-computer interface meeting: Brain-computer interfaces past, present, and future. Brain-Computer Interfaces, 4(1-2), 3-36. https://doi. org/10.1080/2326263X.2016.1275488 
Huggins, J. E., \& Kovacs, T. (2018). Brain-computer interfaces for augmentative and alternative communication: Separating the reality from the hype. Perspectives of the ASHA Special Interest Groups, 3(12), 13-23. https://doi. org/10.1044/persp3.sig12.13

Huggins, J. E., Wren, P., \& Gruis, K. (2011). What would brain-computer interface users want? Opinions and priorities of potential users with amyotrophic lateral sclerosis. Amyotrophic Lateral Sclerosis, 12(5), 318-324. https://doi. org/10.3109/17482968.2011.572978 Igbaria, M. (1993). User acceptance of microcomputer technology: An empirical test. Omega, 21(1), 73-90. https:// doi.org/10.1016/0305-0483(93)90040-R

Ikegami, S., Takano, K., Saeki, N., \& Kansaku, K. (2011). Operation of a P300based brain-computer interface by individuals with cervical spinal cord injury. Clinical Neurophysiology, 122(5), 991-996. https://doi.org/10.1016/j. clinph.2010.08.021

Johnson, J. M., Inglebret, E., Jones, C., \& Ray, J. (2006). Perspectives of speech language pathologists regarding success versus abandonment of AAC. Augmentative and Alternative Communication, 22(2), 85-99. https://doi. org/10.1080/07434610500483588

Käthner, I., Kübler, A., \& Halder, S. (2015). Comparison of eye tracking, electrooculography and an auditory brain-computer interface for binary communication: A case study with a participant in the locked-in state. Journal of NeuroEngineering and Rehabilitation, 12(1), 76. https://doi.org/10.1186/ s12984-015-0071-z

Kent-Walsh, J., \& Binger, C. (2018). Methodological advances, opportunities, and challenges in AAC research. Augmentative and Alternative Communication, 34(2), 93-103. https://doi.org/10.1080/07434618.2018.1456560

Kilbourne, A. M., Glasgow, R. E., \& Chambers, D. A. (2020). What can implementation science do for you? Key success stories from the field. Journal of General Internal Medicine, 35, 783-787. https://doi.org/10.1007/ s11606-020-06174-6

Kinney-Lang, E., Kelly, D., Floreani, E. D., Jadavji, Z., Rowley, D., Zewdie, E. T., Anaraki, J. R., Bahari, H., Beckers, K., Castelane, K., Crawford, L., House, S., Rauh, C. A., Michaud, A., Mussi, M., Silver, J., Tuck, C., Adams, K., Andersen, J., ... Kirton, A. (2020). Advancing brain-computer interface applications for severely disabled children through a multidisciplinary national network: Summary of the inaugural pediatric BCI Canada meeting. Frontiers in Human Neuroscience, 14, 593883. https://doi.org/10.3389/fnhum.2020.593883.

Kleih, S. C., Gottschalt, L., Teichlein, E., \& Weilbach, F. X. (2016). Toward a P300 based brain-computer interface for aphasia rehabilitation after stroke: Presentation of theoretical considerations and a pilot feasibility study. Frontiers in Human Neuroscience, 10, 547. https://doi.org/10.3389/fnhum.201

Kögel, J., Jox, R. J., \& Friedrich, O. (2020). What is it like to use a BCI?-Insights from an interview study with brain-computer interface users. BMC Medical Ethics, 21, 2. https://doi.org/10.1186/s12910-019-0442-2 
Kübler, A., Holz, E., Riccio, A., Zickler, C., Kaufmann, T., Kleih, S., Staiger-Sälzer, P., Desideri, L., Hoogerwerf, E., \& Mattia, D. (2014). The user-centered design as novel perspective for evaluating the usability of BCI-controlled applications. PLOS ONE, 9(12), Article e112392. https://doi.org/10.1371/journal. pone.0112392

Light, J., \& McNaughton, D. (2015). Designing AAC research and intervention to improve outcomes for individuals with complex communication needs. Augmentative and Alternative Communication, 31(2), 85-96. https://doi.org/10. 3109/07434618.2015.1036458

Lorenz, R., Pascual, J., Blankertz, B., \& Vidaurre, C. (2014). Towards a holistic assessment of the user experience with hybrid BCIs. Journal of Neural Engineering, 11(3), Article 035007. https://doi. org/10.1088/1741-2560/11/3/035007

Lotte, F., Larrue, F., \& Mühl, C. (2013). Flaws in current human training protocols for spontaneous brain-computer interfaces: Lessons learned from instructional design. Frontiers in Human Neuroscience, 7, 568. https://doi.org/10.3389/ fnhum.2013.00568

Mayaud, L., Congedo, M., Van Laghenhove, A., Orlikowski, D., Figère, M., Azabou, E., \& Cheliout-Heraut, F. (2013). A comparison of recording modalities of $\mathrm{P}_{300}$ event-related potentials (ERP) for brain-computer interface (BCI) paradigm. Neurophysiologie Clinique/Clinical Neurophysiology, 43(4), 217-227. https:// doi.org/10.1016/j.neucli.2013.06.002

Mendel, P., Meredith, L. S., Schoenbaum, M., Sherbourne, C. D., \& Wells, K. B. (2008). Interventions in organizational and community context: A framework for building evidence on dissemination and implementation in health services research. Administration and Policy in Mental Health and Mental Health Services Research, 35(1-2), 21-37. https://doi.org/10.1007/s10488-007-0144-9

Meyers, D. C., Durlak, J. A., \& Wandersman, A. (2012). The quality implementation framework: A synthesis of critical steps in the implementation process. American Journal of Community Psychology, 50(3-4), 462-480. https://doi. org/10.1007/s10464-012-9522-X

Moir, T. (2018). Why is implementation science important for intervention design and evaluation within educational settings? Frontiers in Education, 3, 61. https://doi.org/10.3389/feduc.2018.00061

Nilsen, P. (2015). Making sense of implementation theories, models and frameworks. Implementation Science, 10, 53. https://doi.org/10.1186/ S13012-015-0242-0

Nilsen, P., \& Bernhardsson, S. (2019). Context matters in implementation science: A scoping review of determinant frameworks that describe contextual determinants for implementation outcomes. BMC Health Services Research, 19(1), 189. https://doi.org/10.1186/s12913-019-4015-3

Ogden, T., \& Fixsen, D. L. (2014). Implementation Science. Zeitschrift für Psychologie, 222(1), 4-11. https://doi.org/10.1027/2151-2604/ao00160 
Olswang, L., \& Prelock, P. (2015). Bridging the gap between research and practice: Implementation science. Journal of Speech, Language, and Hearing Research, 58(6), S1818-S1826. https://doi.org/10.1044/2015 JSLHR-L-14-0305

Pasqualotto, E., Matuz, T., Federici, S., Ruf, C. A., Bartl, M., Olivetti Belardinelli, M., Birbaumer, N., \& Halder, S. (2015). Usability and workload of access technology for people with severe motor impairment: A comparison of braincomputer interfacing and eye tracking. Neurorehabilitation and Neural Repair, 29(10), 950-957. https://doi.org/10.1177/1545968315575611

Peters, B., Bieker, G., Heckman, S. M., Huggins, J. E., Wolf, C., Zeitlin, D., \& Fried-Oken, M. (2015). Brain-computer interface users speak up: The virtual users' forum at the 2013 international brain-computer interface meeting. Archives of Physical Medicine and Rehabilitation, 96(3), S33-S37. https://doi. org/10.1016/j.apmr.2014.03.037

Peters, B., Mooney, A., Oken, B., \& Fried-Oken, M. (2016). Soliciting BCI user experience feedback from people with severe speech and physical impairments. Brain-Computer Interfaces, 3(1), 47-58. https://doi.org/10.1080/23262 63X.2015.1138056

Peters, D. H., Adam, T., Alonge, O., Agyepong, I. A., \& Tran, N. (2013). Implementation research: What it is and how to do it. BMJ, 347, 731-736. https://doi.org/10.1136/bmj.f6753

Pitt, K. M., \& Brumberg, J. S. (2018a). A screening protocol incorporating braincomputer interface feature matching considerations for augmentative and alternative communication. Assistive Technology, 32(3), 161-172. https://doi.or $\mathrm{g} / 10.1080 / 10400435.2018 .1512175$

Pitt, K. M., \& Brumberg, J. S. (2018b). Guidelines for feature matching assessment of brain-computer interfaces for augmentative and alternative communication. American Journal of Speech-Language Pathology, 27(3), 950-964. https://doi. org/10.1044/2018 ajslp-17-013 5

Pitt, K. M., \& Brumberg, J. S. (2021a). Evaluating person-centered factors associated with brain-computer interface access to a commercial augmentative and alternative communication paradigm. Assistive Technology, 1-10. https:// doi.org/10.1080/10400435.2021.1872737

Pitt, K. M., \& Brumberg, J. S. (2021b). Evaluating the perspectives of those with severe physical impairments while learning BCI control of a commercial augmentative and alternative communication paradigm. Assistive Technology, 1-9. https://doi.org/10.1080/10400435.2021.1949405

Pitt, K. M., Brumberg, J. S., Burnison, J. D., Mehta, J., \& Kidwai, J. (2019). Behind the scenes of noninvasive brain- computer interfaces: A review of electroencephalography signals, how they are recorded, and why they matter. Perspectives of the ASHA Special Interests Groups, 4(6), 1622-1636. https://doi. org/10.1044/2019_PERS-19-00059

Pitt, K. M., Brumberg, J. S., \& Pitt, A. R. (2019). Evaluating person-centered factors associated with brain-computer interface access to a commercial augmentative and alternative communication paradigm. Assistive Technology 
Outcomes and Benefits, 13, 1-10. https://doi.org/10.1080/10400435.2021.1872

737

Pouplin, S., Roche, N., Vaugier, I., Cabanilles, S., Hugeron, C., \& Bensmail, D. (2016). Text input speed in persons with cervical spinal cord injury. Spinal Cord, 54(2), 158-162. https://doi.org/10.1038/sc.2015.147

Proctor, E., Silmere, H., Raghavan, R., Hovmand, P., Aarons, G., Bunger, A., Griffey, R., \& Hensley, M. (2011). Outcomes for implementation research: Conceptual distinctions, measurement challenges, and research agenda. Administration and Policy in Mental Health, 38(2), 65-76. https://doi. org/10.1007/s10488-010-0319-7

Rosenberg, R. N. (2003). Translating biomedical research to the bedside: A national crisis and a call to action. JAMA, 289(10), 1305-1306. https://doi. org/10.1001/jama.289.10.1305

Rosenberg, S., \& Beukelman, D. (1987). The Participation Model. In C. A. Coston (Ed.), Proceedings of the national planners conference on assistive device service delivery (pp. 159-161). The Association for the Advancement of Rehabilitation Technology.

Saha, S., Mamun, K. A., Ahmed, K., Mostafa, R., Naik, G. R., Darvishi, S., Khondaker, A. H., \& Baumert, M. (2021). Progress in brain computer interface: Challenges and opportunities. Frontiers in Systems Neuroscience, 15, 578875. https://doi.org/10.3389/fnsys.2021.578875

Scherer, R., Billinger, M., Wagner, J., Schwarz, A., Hettich, D. T., Bolinger, E., Garcia, M. L., Navarro, J., \& Müller- Putz, G. (2015). Thought-based row-column scanning communication board for individuals with cerebral palsy. Annals of Physical and Rehabilitation Medicine, 58(1), 14-22. https://doi.org/10.1016/j. rehab.2014.11.005

Shahriari, Y., Vaughan, T. M., McCane, L. M., Allison, B. Z., Wolpaw, J. R., \& Krusienski, D. J. (2019). An exploration of BCI performance variations in people with amyotrophic lateral sclerosis using longitudinal EEG data. Journal of Neural Engineering, 16(5), Article 056031. https://doi.org/10.1088/1741-2552/ ab22ea

Shenoy, P., Krauledat, M., Blankertz, B., Rao, R., \& Müller, K. (2006). Towards adaptive classification for BCI. Journal of Neural Engineering, 3(1), R13-R23. https://doi.org/10.1088/1741-2560/3/1/ro2

Stevens, E. R., Shelley, D., \& Boden-Albala, B. (2020). Perceptions of barriers and facilitators to engaging in implementation science: A qualitative study. Public Health, 185, 318-323. https://doi.org/10.1016/j.puhe.2020.06.016

Talukdar, U., Hazarika, S. M., \& Gan, J. Q. (2019). Motor imagery and mental fatigue: Inter-relationship and EEG based estimation. Journal of Computational Neuroscience, 46, 55-76. https://doi.org/10.1007/s10827-018-0701-0

Taylor, M. J., McNicholas, C., Nicolay, C., Darzi, A., Bell, D., \& Reed, J. E. (2014). Systematic review of the application of the plan-do-study-act method to improve quality in healthcare. BMJ Quality \& Safety, 23(4), 290-298. https:// doi.org/10.1136/bmjqs-2013-001862 
Thompson, D. E., Quitadamo, L. R., Mainardi, L., Laghari, K., Gao, S., Kindermans, P.-J., Simeral, J. D., Fazel-Rezai, R., Matteucci, M., Falk, T. H., Bianchi, L., Chestek, C. A., \& Huggins, J. E. (2014). Performance measurement for brain-computer or brain-machine interfaces: A tutorial. Journal of Neural Engineering, 11(3), Article 035001. https://doi. org/10.1088/1741-2560/11/3/035001

Tohidast, S., Mansuri, B., Bagheri, R., \& Azimi, H. (2020). Provision of speechlanguage pathology services for the treatment of speech and language disorders in children during the COVID-19 pandemic: Problems, concerns, and solutions. International Journal of Pediatric Otorhinolaryngology, 138, 110262. https://doi.org/10.1016/j.ijporl.2020.110262

Warren, B., \& Randolph, A. B. (2019). Facebrain: A P30o BCI to Facebook. In F. D. Davis, R. Riedl, J. von Brocke, P.-M. Léger, \& A. B. Randolph (Eds.), Information systems and neuroscience: NeuroIS Retreat 2018 (pp. 119-124). Springer. https://doi.org/10.1007/978-3-030-01087-4 14

Wolpaw, J. R., Bedlack, R. S., Reda, D. J., Ringer, R. J., Banks, P. G., Vaughan, T. M., Heckman, S. M., McCane, L. M., Carmack, C. S., Winden, S., McFarland, D. J., Sellers, E. W., Shi, H., Paine, T., Higgins, D. S., Lo, A. C., Patwa, H. S., Hill, K. J., Huang, G. D., \& Ruff, R. L. (2018). Independent home use of a brain-computer interface by people with amyotrophic lateral sclerosis. Neurology, 91(3), e258e267. https://doi.org/10.1212/wnl.0000000000005812

World Health Organization. (2002). Towards a common language for functioning, disability and health: ICF. https://www.who.it/classifications/icf/ icfbeginnersguide.pdf

Yorkston, K., \& Baylor, C. (2019). Patient-reported outcomes measures: An introduction for clinicians. Perspectives of the ASHA Special Interest Groups, 4(1), 8-15. https://doi.org/10.1044/2018 PERS-ST-2018-0001

Zhang, J., Jadavji, Z., Zewdie, E., \& Kirton, A. (2019). Evaluating if children can use simple brain computer interfaces. Frontiers in Human Neuroscience, 13, 24. https://doi.org/10.3389/fnhum. 2019.00024 